\title{
The evolving role of ribosomes in the regulation of protein synthesis
}

\author{
Karolina Gościńska ${ }^{\square}$ and Ulrike Topf \\ Institute of Biochemistry and Biophysics, Polish Academy of Sciences, Department of Genetics, Warszawa, Poland
}

\begin{abstract}
Maintenance of the cellular homeostasis is firmly linked with protein synthesis. Therefore, it is tightly controlled at multiple levels. An advancement in quantitative techniques, mainly over the last decade, shed new light on the regulation of protein production, which pointed the ribosome as a new player. Ribosomes are macromolecular machines that synthesize polypeptide chains using mRNA as a template. The enormous complexity of ribosomes provides many possibilities of changes in their composition and consecutively in their target specificity. However, it is not clear how this specialization is enforced by the cell and which stimuli provoke that diversity. This review presents an overview of currently available knowledge about ribosome heterogeneity, focusing on changes in protein composition, and their role in the control of translation specificity. Importantly, besides the potential advantage of ribosome-mediated regulation of protein synthesis, its failure can play a crucial role in disease development.
\end{abstract}

Key words: ribosome, translation regulation, ribosome specialization, heterogeneous ribosomes

Received: 05 June, 2020; revised: 08 September, 2020; accepted: 09 September, 2020; available on-line: 27 November, 2020

$\nabla$ e-mail: kgoscinska@ibb.waw.pl

Acknowledgments of Financial Support: This work was supported by the National Science Centre (Poland) grant 2015/19/B/ NZ1/03444

Abbreviations: RP, ribosomal proteins; RBPs, ribosome binding proteins

\section{INTRODUCTION}

Protein synthesis (translation) is a fundamental cellular process essential to maintain the integrity of the proteome. Cells use complex molecular machineries called ribosomes that translate genetic information into polypeptide chains. Protein production is inevitably linked to cell growth and proliferation. Imbalance in protein production can be detrimental for the cell, destroying cellular protein homeostasis and even leading to cell death (Hipp et al., 2014; Rothman, 2010; Santra et al., 2019). Consequently, protein synthesis is tightly regulated and immediately adjusts to environmental changes. Modulation of translation is one of the essential cellular mechanisms responding to stress conditions, such as heat shock, nutrient deprivation, and an increase in reactive oxygen species (ROS) production (Crawford \& Pavitt, 2019; Harding et al., 2003; Hinnebusch, 2005; Liu \& Qian, 2014; Topf et al., 2018; Wrobel et al., 2015). Generally, cells decrease protein synthesis in response to stress to save energy and prevent overproduction of proteins that could overwhelm the capacity of cellular mechanisms protecting from an accumulation of damaged or unfolded proteins (Grant, 2011; Harding et al., 2003; Mohanraj et al., 2020; Topf et al., 2019).

The ribosome is a large ribonucleoprotein machine responsible for synthesizing proteins in all cells using messenger RNA (mRNA) as a template. Eukaryotic ribosomes are comprised of four ribosomal RNA (rRNA) species and 79 ribosomal proteins (RPs) distributed among two distinct subunits together constituting the monosome (80S, named according to the apparent sedimentation velocity) (Thomson et al., 2013). The small ribosomal subunit (40S) is built of $18 \mathrm{~S}$ rRNA and 33 RPs, whereas the large ribosomal subunit (60S) consists of three rRNAs (5S, 5.8S, 25S) and 46 RPs.

The biogenesis of ribosomes takes place within the nucleolus, nucleoplasm, and cytoplasm. In the nucleolus, the RNA polymerase I (Pol I) synthesises 5S, 5.8S, and $18 \mathrm{~S}$ rRNA in a form of a single $47 \mathrm{~S}$ transcript, known as pre-rRNA. The last rRNA, 5S, is transcribed in the nucleus by RNA polymerase III (Pol III). Concomitantly, RNA polymerase II transcribes ribosomal protein-coding genes and the arising mRNA is translated in the cytoplasm. Pre-ribosomal subunits, the $40 \mathrm{~S}$ and 60S, are formed in the nucleolus from processed pre-rRNA, 5S rRNA and ribosomal proteins imported from the cytoplasm. Some of the ribosomal proteins assemble later, after independent export of immature ribosomal subunits to the cytoplasm, including eL24 (L24), eL40 (L40), uL16 (L10) (Fernandez-Pevida et al., 2012; Kruiswijk et al., 1978; Saveanu et al., 2003; Zhou et al., 2019). The size of the mature eukaryotic ribosome is in the range of 3.5 megadaltons (MDa) to 4.0 MDa in higher organisms (Yusupova \& Yusupov, 2017). Although intense studies have been conducted on ribosomes for decades, many questions are still pending, for example, do they consist of the same components in every cell or how ribosome heterogeneity changes the translational output? Here, we discuss the latest findings in the regulatory role of the ribosome in translation, specifically focusing on the role of ribosomal proteins and their modifications.

\section{THE EMERGING CONCEPT OF SPECIALIZED RIBOSOMES}

In 1958, George Palade, who discovered ribosomes, proposed a theory of heterogeneous ribosome particles based on electron microscopy observations of differences in their shape and size (Siekevitz \& Palade, 1958). In parallel, Francis Crick worked on "one gene-one ribosome-one protein hypothesis", according to which the ribosome carries information in its RNA for single protein synthesis (Crick, 1958). Nevertheless, this was disproved by Brenner and colleagues who 
Table 1. Overview of alterations in ribosomes that can contribute to the formation of ribosome specialization. Refer to the review text for details and references.

\begin{tabular}{|c|c|}
\hline Ribosome alterations & Examples \\
\hline \multicolumn{2}{|l|}{ Ribosomal proteins } \\
\hline - Paralogs & uL1b, uL2b, eL8, uL18, eL32, uS4, eS26 \\
\hline - Stoichiometry & uL3, uL18, uL1, eL38, eL40, uS7, eS25, eS26 \\
\hline Post-translational modifications (PTMs) of ribosomal proteins & $\begin{array}{l}\text { acetylation, methylation, glycosylation, phosphorylation, ubiquitina- } \\
\text { tion, oxidation, UFMylation }\end{array}$ \\
\hline PTMs of rRNA & Methylation, Pseudouridylation \\
\hline Ribosome-associated proteins & PKM2, CDK1, BUD23 \\
\hline
\end{tabular}

showed that Escherichia coli ribosomes synthesize phage proteins regardless of infection, demonstrating lack of ribosome specificity (Brenner et al., 1961). For decades, ribosomes were thought to be stable and invariable in composition macromolecular machines which translate available messenger RNA into a protein, however, there is increasing evidence showing their regulatory role (reviewed in Dalla Venezia et al., 2019; Dinman, 2016; Genuth \& Barna, 2018; Xue \& Barna, 2012). In 2002, Mauro and Edelman proposed a ribosome filter hypothesis according to which mRNAs interact differently with ribosomal subunits through changes in their protein composition or rRNA, shedding a light on ribosome control in gene expression (Mauro \& Edelman, 2002). Nowadays, the concept of specialized ribosomes in regulation of translation expands and comprises several layers. Heterogeneity in the ribosome can be determined by differential RP stoichiometry, RP modifications (e.g. phosphorylation, glycosylation), rRNA alterations (e.g. methylation, pseudouridylation) or binding of ribosome-associated proteins (RAPs) (Crawford \& Pavitt, 2019; Shi \& Barna, 2015; Simsek \& Barna, 2017; Sloan et al., 2017; Xue \& Barna, 2012) (Table 1). This variability increases even more when considering interdependent factors in protein synthesis, mRNA and its diversity, such as internal entry sites (IRES), polyA sites in the 3' untranslated region (UTR) or nucleotide modifications (Kozak, 2005; Spriggs et al., 2008). Moreover, ribosome specialization can manifest at the level of a subcellular location, as well as dependence on the cell developmental state, cell type, or even tissue specificity (Guimaraes \& Zavolan, 2016; Gupta \& Warner, 2014; Kondrashov et al., 2011; Marygold et al., 2007; Simsek et al., 2017; Slavov et al., 2015; Wong et al., 2014). Tissue-specific expression was shown for a quarter of human ribosomal proteins (Guimaraes \& Zavolan, 2016). Interestingly, changes in ribosomes affect the translation of a subset of mRNAs rather than global protein synthesis. Perhaps this facilitates responses to urgent cellular protein demand or increases the capability to effectively react to environmental changes, e.g. stress conditions. Nonetheless, the etiology of the variability in ribosome composition and further their regulatory activity remains largely unclear.

The emerging field of ribosome heterogeneity is currently rapidly developing and is driven by implementation of specialized techniques to analyse protein synthesis and ribosomes. However, the findings are scattered and their biological impact touches different fields of cell biology. Thus, we focus on a few recently outstanding findings that highlight modulation of ribosomes at the level of ribosomal proteins themselves and the ribosome - associated proteins (Table 1).

\section{RIBOSOMAL PROTEIN PARALOGS AND DIFFERENTIAL PROTEIN STOICHIOMETRY IN THE RIBOSOME}

The existence of ribosomal protein paralogs has been discovered in many organisms, such as: Arabidopsis thaliana (Barakat et al., 2001; Falcone Ferreyra et al., 2013), the yeasts Saccharomyces cerevisiae and Schizosaccharomyces pombe (Palumbo et al., 2017; Sun et al., 2013), Drosophila melanogaster (Mageeney \& Ware, 2019; Marygold et al., 2007) and in rodents and human cells (Guimaraes \& Zavolan, 2016; Lopes et al., 2010; O'Leary et al., 2013; Sugihara et al., 2010). Among them, the budding yeast appears to be an excellent eukaryotic model for exploring the ribosome heterogeneity. As a result of genome duplication, 59 from 79 RPs of S.cerevisiae are present in paralog pairs with high sequence similarity (Parenteau et al., 2015; Wapinski et al., 2010; Wolfe \& Shields, 1997). Despite their similarity, in most cases deletion of one ribosomal paralog from the pair results in various phenotypes, suggesting their functional diversification ( $\mathrm{Lu}$ et al., 2015; Ni \& Snyder, 2001; Palumbo et al., 2017; Samir et al., 2018; Segev \& Gerst, 2018). To gain further insight, Ghulam et al. compared the expression of ribosomal protein paralog pairs (Ghulam et al., 2020). Focusing on RP paralogs with differences in one or more amino acids (37 out of $59 \mathrm{RP}$ paralogs), specifically pairs producing proteins that could be reproducibly distinguished by applied peptide-based mass-spectrometry (23 pairs). In 20 cases, under normal growth conditions, one of the RP paralogs was predominantly produced (major copy), regardless of their A or B nomenclature. A striking difference was observed for uS4 (S9), where the B paralog represents over $80 \%$ of total protein expression. Interestingly, exposure to stress, such as hygromycin or $\mathrm{NaCl}$, reduced or reversed the ratio of the generated $\mathrm{RP}$ paralogs in most cases, favouring expression of the minor gene copy. Overall genome-wide ChIP-seq study showed no correlation between RNA polymerase II association and the amount of produced ribosomal protein, indicating post-transcriptional regulation of ribosomal protein paralog production (Ghulam et al., 2020). A more in-depth study that used RNA-sequencing and polyribosome association, revealed the importance of RNA abundance in the preferential translation of certain RP paralogs, mainly caused by differences in splicing and 3'end formation (Ghulam et al., 2020). Another study also pointed out the relevance of introns within RPs in control of ribosome function (Parenteau et al., 2011). Altogether this indicates a multilayer regulation of the expression of ribosomal protein paralogs and their distinctive participation in actively translating ribosomes. In response to environmental changes, a subpopulation of the ribosomes that varies in RP paralog occupancy can 


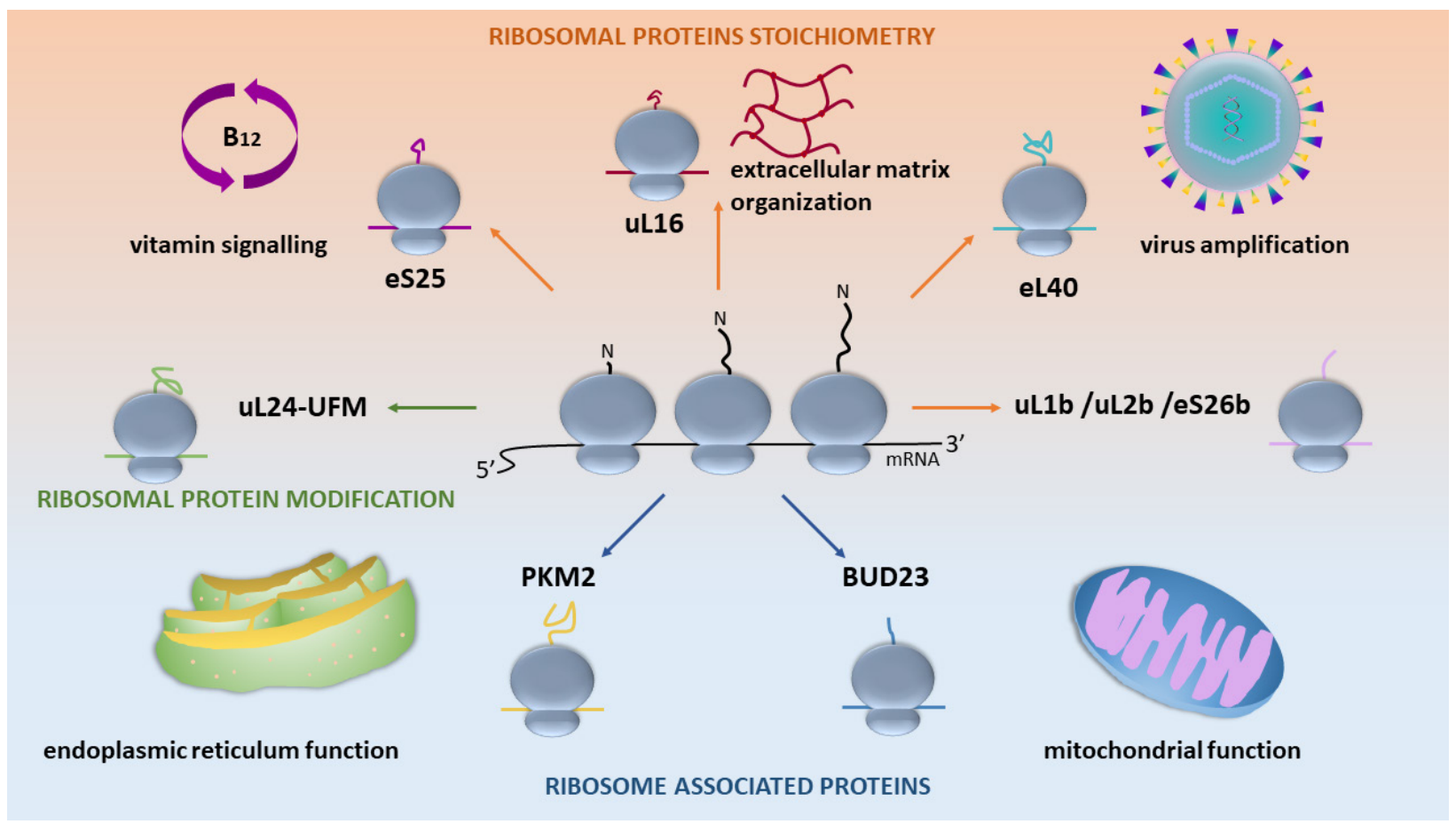

Figure 1. Ribosome heterogeneity regulates translational output.

The variability in translation machinery composition can be manifested at many levels, including differential ribosomal protein stoichiometry, modification of ribosomal proteins or binding of ribosome associated proteins. The subpools of heterogeneous ribosomes arise in response to cellular protein demand and translate distinct mRNAs. The presence of certain ribosomal proteins in the ribosome results in favoured synthesis of proteins involved in vitamin B12 signalling (eS25 (S25)), extracellular matrix organization (uL16 (L10)) or mitochondrial function (uL1b (L1b), uL2b (L2b), eS26b (S26b)). The large ribosomal subunit protein, eL40 (L40), is required for the translation initiation of vesicular stomatitis virus mRNAs. Also, binding of non-ribosomal proteins increases ribosome specialization and results in production of proteins which are necessary for mitochondrial (via BUD23) or endoplasmic reticulum (via PKM2) functions. Moreover, post-translational modification of the ribosomal protein, uL24 (L24) by UFMylation protects from endoplasmic reticulum stress. These examples demonstrate the importance of ribosome heterogeneity for preserving cellular homeostasis.

provide a specific translation output, perhaps to quickly and specifically adapt to a current cellular need.

Functional diversification of RP paralogs can also affect the production of certain groups of the protein. Segev and Gerst showed the relevance of RP paralogs in the translation of mitochondrial proteins (Segev \& Gerst, 2018). The growth of different yeast mutants harbouring deletions of RP paralogs on fermentable (glucose) and non-fermentable (glycerol) carbon sources showed that uL1b (L1b), uL2b (L2b), and eS26b (S26) are necessary under respiratory conditions (Fig. 1). Puromycin-associated nascent chain proteomics (PUNCH-P) technique revealed high downregulation of mitochondrial protein production in these RP mutants. This observation was specific for one RP paralog from the pair, even though they encode an identical protein, such as uL1a (L1a) and $u L 1 b$ (L1b). Notwithstanding, how this specificity is conferred remains unknown.

Concurrently, the eL8 (L8) paralog switch in the 80S ribosome was reported in response to shift in the carbon source from glucose to glycerol, in a yeast culture (Samir et al., 2018). eL8b (L8b) supports cell doubling under respiratory conditions in contrast to eL8a (L8a), confirming no interchangeable functions of RPs paralogs. Moreover, isobaric tags for relative and absolute quantitation (iTRAQ) labelling and mass spectrometry-based quantitative proteomics showed an imbalance in singlecopy ribosomal protein production upon changes in growth conditions, e.g. uL3 (L3) and uL18 (L5), which in turn refers to the new concept of sub-stoichiometric composition of the ribosome (Samir et al., 2018). Recent advances in quantitative techniques also enabled the precise and accurate measurement of ribosome component stoichiometry. In contrast to the uniform ribosome dogma, many studies prove the existence of several heterogeneous subpopulations of translation machinery in the cell at the same time (Shi et al., 2017; Slavov et al., 2015). The appearance of such distinction is usually related to physiological conditions or tissue type. Substantial progress on ribosome stoichiometry was done based on research on embryonic stem cells (Kondrashov et al., 2011; Rao et al., 2012; Shi et al., 2017; Slavov et al., 2015). Shi and others (Shi et al., 2017) applied Selected Reaction Monitoring (SRM) to identify compositions of actively translating ribosomes in mouse embryonic stem cells (mESC). Absolute quantification of 15 RPs in the polysome fractions showed four core RPs to be significantly depleted (uS7 (S5), eS25 (S25), uL1 (L10A), and eL38 (L38)). Also, uL1 (L10A) and eS25 (S25) associate with certain sub-pools of transcripts, e.g. encoding extracellular matrix proteins or involved in B12 vitamin signalling, respectively (Fig. 1). Hence, mESC ribosomes lacking one or more of RPs can still actively engage in the protein synthesis.

Other studies focus on sub-stoichiometry in ribosome composition as an effect of dynamic conditions, like cellular stress. In yeast, high $\mathrm{pH}$ or high salt concentrations lead to the production of eS26 (S26)-depleted ribosomes (Ferretti et al., 2017). Indeed, translatome examination revealed that eS26 (S26)-deficient ribosomes preferentially translate mRNAs implicated in the stress response. Conversely, eS26 (S26)-containing ribosomes occupy transcripts related to the translation process recognizing the Kozak sequence elements. Thus, this work illustrates 
the enhancement of well-known transcriptional stress response at the level of translation which is mediated by the ribosome heterogeneity.

Finally, some RPs exhibit more specialized functions. By studying vesicular stomatitis virus (VSV) protein synthesis upon host shutoff, Lee and others (Lee et al., 2013) discovered that the $60 \mathrm{~S}$ ribosomal subunit protein, eL40 (L40) is needed for translation initiation, in particular, 80S formation on viral mRNAs (Fig. 1) (Fernandez-Pevida et al., 2012). Deep sequencing of polysome associated-mRNA in a yeast model showed eL40 (L40)-depended transcript selectivity, mainly involved in the stress response. Surprisingly, the depletion of eL40 (L40) did not influence the bulk of mRNA - polysome associations $(93 \%)$, confirming the remarkable specialization of eL40 (L40)-bound ribosomes.

\section{RIBOSOMAL PROTEIN MODIFICATIONS}

Ribosome's mode of action can be also changed by RPs' modifications. Such protein modifications include acetylation, methylation, glycosylation, phosphorylation and ubiquitination (Filipovska \& Rackham, 2013; Simsek \& Barna, 2017; Xue \& Barna, 2012). Recently, thiol oxidation and UFMylation are getting increased attention (Shcherbik \& Pestov, 2019; Simsek et al., 2017; Topf et al., 2018; Wang et al., 2020). One of important mechanisms controlling protein function is reversible oxidation and reduction of its cysteine (Cys) residues. An advanced quantitative redox proteomics technique, oxidative isotope-coded affinity tags (OxICAT), revealed several RPs from both ribosome subunits to be ROS sensitive in different organisms (Leichert et al., 2008; Menger et al., 2015; Topf et al., 2018). The SILAC-iodoTMT method, which allows to simultaneously monitor the redox state and protein expression level, also confirms RPs to be one of the most significant groups of proteins affected by the hydrogen peroxide treatment (Vajrychova et al., 2019). Topf et al. proposed a concept that RPs can serve as redox sensors upon oxidative stress conditions (Topf et al., 2018). Dysfunction of mitochondrial protein import results in increased ROS production and rapid attenuation of global protein synthesis. On the other hand, inhibition of protein production caused by exogenous addition of hydrogen peroxide can be reversed by removing the source of stress, suggesting quick translational reprogramming. OxICAT analysis upon mitochondrial stress and exogenous hydrogen peroxide treatment identified overlapping ROS-sensitive ribosomal proteins. Importantly, global translation upon deletions of these proteins was less affected under oxidative stress. Altogether, this renders RPs potential thiol-based redox switches, which might effectively respond to environmental changes. Such a molecular mechanism could be beneficial for the cell, allowing for immediate protein synthesis regulation, without energy-consuming de novo ribosome assembly. Nevertheless, it is still not clear how oxidation of certain RPs influences the work of translation machinery and whether the main outcome of this event is attenuation of the process or rather its reprogramming. Interestingly, other redoxome studies in yeast and also in human cells show that some of the RPs are oxidized under normal growth conditions and play a role in functional pathways (Go et al., 2011; Le Moan et al., 2006). This supports the importance of reversible changes in ribosomal protein redox state in preserving cellular homeostasis.

UFMylation is one of the latest identified ribosomal protein modifications. Ubiquitin-fold modifier 1 (UFM1) is an ubiquitin-like small protein identified in metazoans, plants, and mammals, but not in yeast (Wei \& Xu, 2016). It conjugates to lysine residues on substrates via a specific cascade of enzymatic processes that involve ligases and proteases (Wei \& Xu, 2016). The role of UFM1 is expanding, showing mostly its significance in the unfolded stress response (UPR) in the endoplasmic reticulum (ER) and haematopoiesis (Cai et al., 2016; Lemaire et al., 2011; Tatsumi et al., 2011; Wang et al., 2020; Zhang \& Xu, 2016). An intricate study of Walczak et al., involving UFMylome and MS analysis, revealed that the primary target of UFM1 posttranslational modification is the large ribosomal subunit protein, uL24 (L26), and particularly its C-terminal lysines which are localized next to the ribosome's peptide exit tunnel (Walczak et al., 2019). UFMylated uL24 (L26) was found to be enriched at the ER membrane-bound ribosomes and polysomes, which correlates with the fact that the enzyme complexes catalysing UFMylation and de-UFMylation bind to the ER cytoplasmic surface. Moreover, disruptions of uL24 (L26) UFMylation result in ER stress (Fig. 1). Exploring the function of UFMylation of uL24 (L26) in ER-associated ribosomes, Wang et al. uncovered its implication in the degradation of stalled nascent chains (Wang et al., 2020). Ribosome UFMylation triggers degradation of ER translocation-arrested proteins directing them to the lysosome, in contrast to previously described ribosomeassociated quality control (RQC) mechanism, which targets nascent chains for proteasomal degradation (Brandman \& Hegde, 2016). Thus, modifications of ribosomal proteins can occur at any level of protein production and can have a prominent impact not only on translation regulation but also on the entailed cellular processes.

\section{THE ACTIVITY OF RIBOSOME-ASSOCIATED PROTEINS}

Binding of non-canonical proteins to the ribosome can also contribute to the increase in the diversity of the translational output. A ribo-interactome study performed in mammalian cells revealed hundreds of proteins associating with ribosomes that belong to different functional groups, including protein- and RNAmodifying enzymes, RNA binding proteins, but also proteins involved in the energy metabolism, redox homeostasis and cell cycle (Simsek et al., 2017). Interestingly, a subcellular pool of translating ribosomes interacting with the muscle pyruvate kinase 2 (PKM2) was identified. PKM2 converts phosphoenolpyruvate (PEP) and adenosine diphosphate (ADP) to pyruvate and adenosine triphosphate (ATP) in the last step of glycolysis. This metabolic enzyme appeared to act as a translation activator regardless of its catalytic activity. To find direct PKM2 and RNA interactions, three techniques were combined: ultraviolet (UV) crosslinking, immunoprecipitation (IP), and high-throughput sequencing, together known as the iCLIP analysis. This comprehensive study uncovered that PKM2 interacts with $18 \mathrm{~S}$ rRNA and 28S rRNA in the proximity to the aminoacyl site (A-site) on the ribosome, where charged t-RNA molecules bind during protein synthesis. Additionally, PKM2 mainly targets mRNAs translated by ER-associated ribosomes, encoding components of the ER itself and cellular membrane proteins (Fig. 1). Potentially, it might couple cell metabolism and proliferation. Notably, other iCLIP studies also revealed metabolic enzymes that can interact with RNA, giving a chance to expand the current hypothesis (Baltz et al., 2012; Castello et al., 2012; Liu et al., 2019). Overall, 
this data shows that ribosome associated proteins could boost translational selectivity of spatially localized $\mathrm{mR}$ NAs.

On the other hand, a more direct interplay between cell proliferation and protein synthesis provides cyclindependent kinase 1 (CDK1), lately shown as a translational activator (Haneke et al., 2020). CDK1 is a conserved, central kinase regulating cell cycle, which was identified among other kinases and phosphatases in the siRNA screen upon the formation of stress granules in human cells (Haneke et al., 2020). Although it was already reported as a protein involved in protein synthesis control during mitosis (Shuda et al., 2015), for the first time its role goes beyond the cell cycle control. By synchronizing or arresting certain phases of cell cycle progression, the authors were able to show a reduction in global protein synthesis upon pharmacological inhibition or knockdown of CDK1 in the HeLa cells, as well as in the primary mouse embryonic fibroblasts (MEFs) (Shuda et al., 2015). Many molecular mechanisms contribute to this effect which is downstream of CDK1, such as phosphorylation of $\mathrm{eIF} 2 \alpha$ or S6K1 signalling (Haneke et al., 2020). It is also important to mention that CDK1 cosediments with the polysome fraction, which was confirmed using a mass spectrometry approach (Simsek et al., 2017). The known substrate of CDK1 is the large ribosomal subunit protein, uL11 (L12), which undergoes phosphorylation upon its activation. Interestingly, uL11 (L12) phosphorylation, in turn, enhances the mitotic protein synthesis program (Imami et al., 2018). Finally, ribosome footprinting (Ribo-Seq) revealed that CDK1 boosts 5'TOP mRNA translation, including RPs. Thus, CDK1 serves as a hub connecting the control of protein synthesis and regulation of cell cycle progression.

Further, several studies implicate a role of ribosome heterogeneity in production of mitochondrial proteins (Crawford \& Pavitt, 2019; Segev \& Gerst, 2018). Recently, Baxter and others (Baxter et al., 2020) identified the ribosome associated protein, BUD23, belonging to this group. BUD23 methyltransferase plays a dual, independent role in the formation of the translation apparatus. It is involved in the processing of pre-18S rRNA and adding of methyl mark on its key guanosine residue located within the E site, where deacylated tRNA exits the ribosome and peptidyl-tRNA site (P-site) of the 40S ribosomal subunit. Loss of BUD23 does not significantly affect the global protein production but causes an imbalance in translation of specific mRNA. Translational efficiency (TE) of certain transcripts was calculated as a proportion between 'heavy' polysome fractions, representing more than three ribosomes loaded on one mRNA, and 'light' (less than three). Analysis of combined TE and RNA-seq results showed that BUD23-ribosomes preferentially bind to mRNA transcripts with modest 5'UTR GC content. Surprisingly, depletion of BUD23 impairs the synthesis of nuclear-encoded mitochondrial proteins, especially those comprising complex I, IV, and V of the electron transport chain (Fig. 1). In turn, this greatly diminishes generation of mitochondrial ATP. Ribosomes with associated BUD23 were shown to be necessary for maintaining mitochondrial function, both in vitro and in vivo. In genetically modified mice models, loss of BUD23 has led to embryo-lethality, whereas BUD23 loss restricted to mitochondrial-dependent cells, cardiomyocytes, caused cardiomyopathy and pre-mature death of animals. These findings exemplify the high importance of extrinsic proteins modifying ribosomes to regulate protein synthesis and its impact on organismal physiology.

\section{RIBOSOME MODIFICATION-RELATED DISEASES}

Ribosomopathies are inherited diseases originating from dysfunction of ribosomes caused by mutations in ribosomal genes or rRNA, which give a wide spectrum of clinical phenotypes. However, this definition broadens as a consequence of heterologous ribosomes discoveries. In 1999, the Diamond-Blackfan Anemia (DBA), a bone marrow failure syndrome, was identified as the first disease caused by ribosomal protein mutation, in particular eS19 (S19), thereby supporting the concept of ribosome heterogeneity (Draptchinskaia et al., 1999). The list of DBA related RPs mutations was extended and now involves RPs from both ribosomal subunits, e.g. eS17 (S17), uL18 (L5), and additionally, changes in rRNA were reported (Farrar et al., 2011; Gazda et al., 2008; Lezzerini et al., 2020; Quarello et al., 2016). Ribosomopathies in most cases manifest themselves as hematopoietic deficiencies, and perhaps this is connected to the high diversity in RPs' expression observed in the primary hematopoietic cells. An intriguing phenomenon in ribosomopathies is the transition from hypo- to hyper-proliferation phenotypes, introduced by William Dameshek in 1967 (Dameshek, 1967). Patients who suffer from diseases characterized by a diminished proliferation potential, such as anaemia, with time are at a higher risk of developing hyper-proliferative diseases, such as cancer (Dameshek, 1967; De Keersmaecker, Sulima, Dinman, 2015).

Protein synthesis also plays a crucial role in cancer progression. Cancer cells are characterized by a high proliferative potential and therefore increased mRNA translation demand. Mutations of RPs are linked to several human cancer types, including e.g. breast cancer and T-cell acute lymphoblastic leukaemia (De Keersmaecker et al., 2015; Ferreira et al., 2014; Kampen et al., 2019; Rao et al., 2012). Nevertheless, the so-called oncogenic ribosomes are also characterized by changes in protein composition. One of the newly discovered cancer-related proteins involved in translational reprogramming is the FK506-binding protein 10 (FKBP10) (Ramadori et al., 2020). It is specifically expressed in lung cancer cells and its presence is negatively correlated with the patients' survival. FKBP10 is an ER chaperone with peptidylprolyl-cis-trans-isomerase (PPIase) activity (Chen et al., 2017). Depletion of FKBP10 in A549 cells reduces global protein synthesis by half. Polysome profiling, followed by western blot analysis, detected FKBP10 in the monosome (the 80S) and in a light fraction of polysomes. Further, knockdown of FKBP10 in A549 cells and in vivo in lung tumours in mice models showed accumulation of FKBP10 in the monosome fraction together with a decrease in the polysomes, suggesting its implication in translation elongation. Importantly, upon these conditions ribosome occupancy specifically increases at the proline codons. These results indicate a mechanism relying on the ribosome binding protein, FKBP10, adapted by cancer cells to support their growth by an increase in the protein synthesis. Hence, this could be a promising target for new anticancer therapy (Liang et al., 2019).

Yet, emerging issues are neurodevelopmental syndromes caused by mutations in the ribosomal machinery's components or association of trans-acting proteins, e.g. intellectual retardation and schizophrenia (Zhou et al., 2018). Several studies report mutations in the uL16 (L10) gene which gives different phenotypes depending on localization of the mutation (Brooks et al., 2014). Mutation at the N-terminus of the uL16 (L10) protein results in inter alia microcephaly, whereas at C-terminus 
correlates with autism (Brooks et al., 2014). Knockdown of uL16 (L10) in zebrafish gives a microcephaly-like phenotype and results in a decrease in general protein synthesis in the brain, which could not be rescued by mutated uL16 (L10) identified in patients (Brooks et al., 2014). This proves the role of uL16 (L10) in symptoms developed by affected individuals, but the exact mechanism remains unknown. Similarly, protein synthesis dysregulation was observed in patients with developmental delay carrying a mutation in a small ribosomal protein, uS12 (S23) (Paolini et al., 2017). Corresponding mutations in yeast show impairment in accuracy of mRNA translation, while global protein synthesis remains unchanged, most likely meaning that mutated uS12 (S23) is an integral part of translational machinery (Paolini et al., 2017). Noteworthy, fibroblasts from affected patients display high sensitivity to oxidative stress (Paolini et al., 2017). In conclusion, changes in ribosomes are the cause of many distinct pathological phenotypes implicated in a wide spectrum of diseases, thus making them an attractive treatment targets, as well as potential disease prognosis markers.

\section{CONCLUSIONS AND FURTHER PERSPECTIVES}

Protein synthesis is a crucial biological process in which deregulation has a tremendous impact on the cellular or even organismal fitness. The discovery of ribosome heterogeneity is a milestone in understanding of gene expression. Advancement in molecular and analytical techniques, such as mass spectrometry, paves the way towards deciphering ribosome functions in the control of protein production (Genuth \& Barna, 2018; Samir et al., 2018; Simsek et al., 2017; Topf et al., 2018). Multiple high-throughput analyses allow for identification of a vast number of alterations in the translation machinery, such as diversity in protein composition, changes in rRNA structure, or association of extrinsic proteins. The ribosome is an intricate ribonucleoprotein complex and for this reason it can also be a source of countless modifications, which in turn renders its study challenging. Generation of specialized ribosome sub-populations is usually linked to development, the status of the cell, or environmental conditions, such as stress. Pieces of evidence also suggest ribosome differentiation according to cell type or tissue specificity (Guimaraes \& Zavolan, 2016; Kondrashov et al., 2011; Wong et al., 2014). A seemingly subtle change, a single variation in the translation machinery can modulate translational output or even leads to a phenotypic effect in the whole organism. Likewise, such diversity also increases the risk of dysfunctional ribosomes' formation or production of redundant proteins, which can disturb cell homeostasis. In the context of diseases, the finding of specialized ribosomes results in a more detailed understanding of certain molecular mechanisms and in consequence the pathophysiology of human diseases (Tahmasebi et al., 2018). Importantly, this opens new perspectives for targeted therapies. As already mentioned, a single modification in the translation machinery can cause a wide range of symptoms, proving its enormous impact on protein synthesis and further organismal health and vitality. Altogether, occurrence of ribosome heterogeneity opens many questions. Among them are the existence of signals for the generation of new ribosome subpopulations, what determines their localization, or if their appearance changes with aging? Currently, available knowledge regarding ribosome heterogeneity and its regulatory role in gene expression superficially touches different fields and is lacking consistency. There is a need for more systematic studies and identification of functional consequences to further understand its implication in physiology and pathophysiology.

\section{REFERENCES}

Baltz AG, Munschauer M, Schwanhausser B, Vasile A, Murakawa Y, Schueler M, Youngs N, Penfold-Brown D, Drew K, Milek M, et al (2012) The mRNA-bound proteome and its global occupancy profile on protein-coding transcripts. Mol Cell 46: 674-690. https://doi. org/10.1016/j.molcel.2012.05.021

Barakat A, Szick-Miranda K, Chang IF, Guyot R, Blanc G, Cooke R, Delseny M, Bailey-Serres J (2001) The organization of cytoplasmic ribosomal protein genes in the Arabidopsis genome. Plant Physiol 127: 398-415

Baxter M, Voronkov M, Poolman T, Galli G, Pinali C, Goosey L, Knight A, Krakowiak K, Maidstone R, Iqbal M, Zi M, Prehar S, Cartwright EJ, Gibbs J, Matthews LC, Adamson AD, Humphreys NE, Rebelo-Guiomar P, Minczuk M, Bechtold DA, Loudon A, Ray D (2020) Cardiac mitochondrial function depends on BUD23 mediated ribosome programming. Elife 9. https://doi.org/10.7554/ eLife. 50705

Ben-Sahra I, Manning BD (2017) mTORC1 signaling and the metabolic control of cell growth. Curr Opin Cell Biol 45 72-82. https:// doi.org/10.1016/i.ceb.2017.02.012

Brandman O, Hegde RS (2016) Ribosome-associated protein quality control. Nat Struct Mol Biol 23: 7-15. https://doi.org/10.1038/ nsmb. 3147

Brenner S, Jacob F, Meselson M (1961) An unstable intermediate carrying information from genes to ribosomes for protein synthesis. Nature 190 576-581. https://doi.org/10.1038/190576a0

Brooks SS, Wall AL, Golzio C, Reid DW, Kondyles A, Willer JR, Botti C, Nicchitta CV, Katsanis N, Davis EE (2014) A novel ribosomopathy caused by dysfunction of RPL10 disrupts neurodevelopment and causes X-linked microcephaly in humans. Genetics 198: 723-733. https://doi.org/10.1534/genetics.114.168211

Cai YF, Singh N, Li HL (2016) Essential role of Ufm1 conjugation in the hematopoietic system. Exp Hematol 44: 442-446. https://doi. org/10.1016/j.exphem.2016.03.007

Castello A, Fischer B, Eichelbaum K, Horos R, Beckmann BM, Strein C, Davey NE, Humphreys DT, Preiss T, Steinmetz LM, Krijgsveld J, Hentze MW (2012) Insights into RNA biology from an atlas of mammalian mRNA-binding proteins. Cell 149: 1393-1406. https:// doi.org/10.1016/j.cell.2012.04.031

Chen Y, Terajima M, Banerjee P, Guo H, Liu X, Yu J, Yamauchi M, Kurie JM (2017) FKBP65-dependent peptidyl-prolyl isomerase activity potentiates the lysyl hydroxylase 2 -driven collagen cross-link switch. Sci Rep 7 46021. https://doi.org/10.1038/srep46021

Costa-Mattioli M, Walter P (2020) The integrated stress response: From mechanism to disease. Science 368(6489). https://doi.org/10.1126/ science.aat 5314

Crawford RA, Pavitt GD (2019) Translational regulation in response to stress in Saccharomyces cerevisiae. Yeast 36: 5-21. https://doi. org/10.1002/yea.3349

Crick FH (1958) On protein synthesis. Symp Soc Exp Biol 12 138-163

Dalla Venezia N, Vincent A, Marcel V, Catez F, Diaz J. J (2019) Emerging role of eukaryote ribosomes in translational control. Int J Mol Sci 20: https://doi.org/10.3390/ijms20051226

Dameshek W (1967) Riddle - What do aplastic anemia paroxysmal nocturnal hemoglobinuria (Pnh) and hypoplastic leukemia have in common. Blood 30: 251-254

De Keersmaecker K, Sulima SO, Dinman JD (2015) Ribosomopathies and the paradox of cellular hypo- to hyperproliferation. Blood 125: 1377-1382. https://doi.org/10.1182/blood-2014-10-569616

Dever TE, Dinman JD, Green R (2018) Translation elongation and recoding in eukaryotes. Cold Spring Harb Perspect Biol 10: https://doi. org/10.1101/cshperspect.a032649

Dinman JD (2016) Pathways to specialized ribosomes: the brussels lecture. J Mol Biol 428(10 Pt B): 2186-2194. https://doi.org/10.1016/j. jmb.2015.12.021

Donnelly N, Gorman AM, Gupta S, Samali A (2013) The eIF2 alpha kinases: their structures and functions. Cell Mol Life Sci 70: 34933511. https://doi.org/10.1007/s00018-012-1252-6

Draptchinskaia N, Gustavsson P, Andersson B, Pettersson M, Willig T. N, Dianzani I, Ball S, Tchernia G, Klar J, Matsson H, Tentler D, Mohandas N, Carlsson B, Dahl N (1999) The gene encoding ribosomal protein S19 is mutated in Diamond-Blackfan anaemia. Nature Genet 21: 169-175

Falcone Ferreyra ML, Casadevall R, Luciani MD, Pezza A, Casati P (2013) New evidence for differential roles of 110 ribosomal proteins from Arabidopsis. Plant Physiol 163: 378-391. https://doi. org/10.1104/pp.113.223222 
Farrar JE, Vlachos A, Atsidaftos E, Carlson-Donohoe H, Markello TC, Arceci RJ, Ellis SR, Lipton JM, Bodine DM (2011) Ribosomal protein gene deletions in Diamond-Blackfan anemia. Blood 118: 69436951. https://doi.org/10.1182/blood-2011-08-375170

Fernandez-Pevida A, Rodriguez-Galan O, Diaz-Quintana A, Kressler D, de la Cruz J (2012) Yeast ribosomal protein L40 assembles late into precursor $60 \mathrm{~S}$ ribosomes and is required for their cytoplasmic maturation. J Biol Chem 287. https://doi.org/10.1074/jbc. M112.400564

Ferreira AM, Tuominen I, van Dijk-Bos K, Sanjabi B, van der Sluis T, van der Zee AG, Hollema H, Zazula M, Sijmons RH, Aaltonen LA, Westers H, Hofstra RM (2014) High frequency of RPL22 mutations in microsatellite - unstable colorectal and endometrial tumors. Hum Mutat 35: 1442-1445. https://doi.org/10.1002/humu.22686

Ferretti MB, Ghalei H, Ward EA, Potts EL, Karbstein K (2017) Rps26 directs mRNA-specific translation by recognition of Kozak sequence elements. Nat Struct Mol Biol 24: 700-707. https://doi. org/10.1038/nsmb.3442

Filipovska A, Rackham O (2013) Specialization from synthesis: How ribosome diversity can customize protein function. FEBS Letters 587: 1189-1197. https://doi.org/10.1016/j.febslet.2013.02.032

Gazda HT, Sheen MR, Vlachos A, Choesmel V, O’Donohue MF, Schneider H, Darras N, Hasman C, Sieff CA, Newburger PE, Ball SE, Niewiadomska E, Matysiak M, Zaucha JM, Glader B, Niemeyer C, Meerpohl JJ, Atsidaftos E, Lipton JM, Gleizes PE, Beggs AH (2008) Ribosomal protein L5 and L11 mutations are associated with cleft palate and abnormal thumbs in Diamond-Blackfan anemia patients. Am J Hum Genet 83: 769-780. https://doi.org/10.1016/j. ajhg.2008.11.004

Genuth NR, Barna M (2018) The discovery of ribosome heterogeneity and its implications for gene regulation and organismal life. Mol Cell 71: 364-374. https://doi.org/10.1016/j.molcel.2018.07.018

Ghulam MM, Catala M, Abou Elela S (2020) Differential expression of duplicated ribosomal protein genes modifies ribosome composition in response to stress. Nucleic Acids Res 48: 1954-1968. https://doi. org/10.1093/nar/gkz1183

Go YM, Duong DM, Peng J, Jones DP (2011) Protein cysteines map to functional networks according to steady-state level of oxidation. $J$ Proteomics Bioinform 4: 196-209. https://doi.org/10.4172/jpb.1000190

Gold VA, Chroscicki P, Bragoszewski P, Chacinska A (2017) Visualization of cytosolic ribosomes on the surface of mitochondria by electron cryo-tomography. EMBO Rep 18: 1786-1800. https://doi. org/10.15252/embr.201744261

Grant CM (2011) Regulation of translation by hydrogen peroxide. Antioxid Redox Signal 15: 191-203. https://doi.org/10.1089/ ars. 2010.3699

Guimaraes JC, Zavolan M (2016) Patterns of ribosomal protein expression specify normal and malignant human cells. Genome Biol 17: 236. https://doi.org/10.1186/s13059-016-1104-z

Gupta V, Warner JR (2014) Ribosome-omics of the human ribosome. RNA 20: 1004-1013. https://doi.org/10.1261/rna.043653.113

Haneke K, Schott J, Lindner D, Hollensen AK, Damgaard C. K, Mongis C, Knop M, Palm W, Ruggieri A, Stoecklin G (2020) CDK1 couples proliferation with protein synthesis. J Cell Biol 219: https:// doi.org/10.1083/jcb.201906147

Harding HP, Zhang Y, Zeng H, Novoa I, Lu PD, Calfon M, Sadri N, Yun C, Popko B, Paules R, Stojdl DF, Bell JC, Hettmann T, Leiden JM, Ron D (2003) An integrated stress response regulates amino acid metabolism and resistance to oxidative stress. Mol Cell 11: 619-633. https://doi.org/10.1016/s1097-2765(03)00105-9

Hinnebusch AG (2005) Translational regulation of GCN4 and the general amino acid control of yeast. Annu Rev Microbiol 59 407-450. https://doi.org/10.1146/annurev.micro.59.031805.133833

Hipp MS, Park SH, Hartl FU (2014) Proteostasis impairment in protein-misfolding and -aggregation diseases. Trends Cell Biol 24: 506514. https://doi.org/10.1016/j.tcb.2014.05.003

Holz MK, Ballif BA, Gygi SP, Blenis J (2005) mTOR and S6K1 mediate assembly of the translation preinitiation complex through dynamic protein interchange and ordered phosphorylation events. Cell 123: 569-580. https://doi.org/10.1016/j.cell.2005.10.024

Imami K, Milek M, Bogdanow B, Yasuda T, Kastelic N, Zauber H, Ishihama Y, Landthaler M, Selbach M (2018) Phosphorylation of the ribosomal protein RPL12/uL11 affects translation during mitosis. Mol Cell 72: 84-98 e89. https://doi.org/10.1016/j.molcel.2018.08.019

Jackson RJ, Hellen CU, Pestova TV (2010) The mechanism of eukaryotic translation initiation and principles of its regulation. Nat Rev Mol Cell Biol 11: 113-127. https://doi.org/10.1038/nrm2838

Kampen KR, Sulima SO, Verbelen B, Girardi T, Vereecke S, Rinaldi G, Verbeeck J, Op de Beeck J, Uyttebroeck A, Meijerink JPP, et al (2019) The ribosomal RPL10 R98S mutation drives IRES-dependent BCL-2 translation in T-ALL. Leukemia 33: 319-332. https://doi. org/10.1038/s41375-018-0176-z

Klinge S, Woolford JL (2019) Ribosome assembly coming into focus. Nat Rev Mol Cell Biol 20: 116-131. https://doi.org/10.1038/s41580018-0078-y
Kondrashov N, Pusic A, Stumpf CR, Shimizu K, Hsieh AC, Ishijima J, Shiroishi T, Barna M (2011) Ribosome-mediated specificity in Hox mRNA translation and vertebrate tissue patterning. Cell 145: 383 397. https://doi.org/10.1016/j.cell.2011.03.028

Kozak M (2005) Regulation of translation via mRNA structure in prokaryotes and eukaryotes. Gene 361 13-37. https://doi.org/10.1016/j.gene.2005.06.037

Kruiswijk T, Planta RJ, Krop JM (1978) The course of the assembly of ribosomal subunits in yeast. Biochim Biophys Acta 517: 378-389. https://doi.org/10.1016/0005-2787(78)90204-6

Kwan T, Thompson SR (2019) Noncanonical translation initiation in eukaryotes. Cold Spring Harb Perspect Biol 11: https://doi. org/10.1101/cshperspect.a032672

Le Moan N, Clement G, Le Maout S, Tacnet F, Toledano MB (2006) The Saccharomyces cerevisiae proteome of oxidized protein thiols - contrasted functions for the thioredoxin and glutathione pathways. J Biol Chem 281: 10420-10430. https://doi.org/10.1074/jbc. M513346200

Leichert LI, Gehrke F, Gudiseva HV, Blackwell T, Ilbert M, Walker AK, Strahler JR, Andrews PC, Jakob U (2008) Quantifying changes in the thiol redox proteome upon oxidative stress in vivo. Proc Natl Acad Sci U S A 105: 8197-8202. https://doi.org/10.1073/ pnas.0707723105

Lemaire K, Moura RF, Granvik M, Igoillo-Esteve M, Hohmeier HE, Hendrickx N, Newgard CB, Waelkens E, Cnop M, Schuit F (2011) Ubiquitin fold modifier 1 (UFM1) and its target UFBP1 protect pancreatic beta cells from ER stress-induced apoptosis. PLoS One 6: e18517. https://doi.org/10.1371/journal.pone.0018517

Lezzerini M, Penzo M, O'donohue MF, Vieira CMD, Saby M, Elfrink HL, Diets IJ, Hesse AM, Coute Y, Gastou M, Nin-Velez A, Nikkels PGJ, Olson AN, Zonneveld-Huijssoon E, Jongmans MCJ, Zhang G, van Weeghel M, Houtkooper RH, Wlodarski MW, Kuiper RP, Bierings MB, van der Werff Ten Bosch J, Leblanc T, Montanaro L, Dinman JD, Da Costa L, Gleizes PE (2020) Ribosomal protein gene RPL9 variants can differentially impair ribosome function and cellular metabolism. Nucleic Acids Res 48: 770-787. https://doi. org/10.1093/nar/gkz1042

Liang L, Zhao K, Zhu JH, Chen G, Qin XG, Chen JQ (2019) Comprehensive evaluation of FKBP10 expression and its prognostic potential in gastric cancer. Oncol Rep 42: 615-628. https://doi. org/10.3892/or.2019.7195

Liu B, Qian SB (2014) Translational reprogramming in cellular stress response. Wiley Interdiscip Rev RNA 5: 301-315. https://doi. org/10.1002/wrna.1212

Liu LC, Li T, Song G, He QX, Yin YF, Lu JYY, Bi XJ, Wang KL, Luo S, Chen YS, Yang Y, Sun BF, Yang YG, Wu J, Zhu H, Shen $\mathrm{X}$ (2019) Insight into novel RNA-binding activities via large-scale analysis of lncRNA-bound proteome and IDH1-bound transcriptome. Nucleic Acids Res 47: 2244-2262. https://doi.org/10.1093/ nar/gkz032

Lopes AM, Miguel RN, Sargent CA, Ellis PJ, Amorim A, Affara NA (2010) The human RPS4 paralogue on Yq11.223 encodes a structurally conserved ribosomal protein and is preferentially expressed during spermatogenesis. Bmc Mol Biol 11. https://doi. org/10.1186/1471-2199-11-33

Lu H, Yao XW, Whiteway M, Xiong J, Liao ZB, Jiang YY, Cao YY (2015) Loss of RPS41 but not its paralog RPS42 results in altered growth filamentation and transcriptome changes in Candida albicans. Fungal Genet Biol 80 31-42. https://doi.org/10.1016/j. fgb.2015.03.012

Mageeney CM, Ware VC (2019) Specialized eRpL22 paralogue-specific ribosomes regulate specific mRNA translation in spermatogenesis in Drosophila melanogaster. Mol Biol Cell 30: 2240-2253. https://doi. org/10.1091/mbc.E19-02-0086

Magnuson B, Ekim B, Fingar DC (2012) Regulation and function of ribosomal protein S6 kinase (S6K) within mTOR signalling networks. Biochem J 441: 1-21. https://doi.org/10.1042/Bj20110892

Mahamid J, Pfeffer S, Schaffer M, Villa E, Danev R, Cuellar LK, Forster F, Hyman AA, Plitzko JM, Baumeister W (2016) Visualizing the molecular sociology at the HeLa cell nuclear periphery. Science 351: 969-972. https://doi.org/10.1126/science.aad8857

Marygold SJ, Roote J, Reuter G, Lambertsson A, Ashburner M, Millburn GH, Harrison PM, Yu Z, Kenmochi N, Kaufman TC, Leevers SJ, Cook KR (2007) The ribosomal protein genes and Minute loci of Drosophila melanogaster. Genome Biol 8: R216. https://doi. org/10.1186/gb-2007-8-10-r216

Mauro VP, Edelman GM (2002) The ribosome filter hypothesis. Proc Natl Acad Sci U S A 99: 12031-12036. https://doi.org/10.1073/ pnas.192442499

Menger KE, James AM, Cocheme HM, Harbour ME, Chouchani ET, Ding SJ, Fearnley IM, Partridge L, Murphy MP (2015) Fasting but not aging dramatically alters the redox status of cysteine residues on proteins in Drosophila melanogaster. Cell Reports 13: 1285-1285. https://doi.org/10.1016/j.celrep.2015.10.048 
Mohanraj K, Nowicka U, Chacinska A (2020) Mitochondrial control of cellular protein homeostasis. Biochem J 477: 3033-3054. https://doi. org/10.1042/BCJ20190654

Morita M, Gravel SP, Hulea L, Larsson O, Pollak M, St-Pierre J, Topisirovic I (2015) mTOR coordinates protein synthesis mitochondrial activity and proliferation. Cell Cycle 14: 473-480. https://doi.org/10. 4161/15384101.2014.991572

Ni L, Snyder M (2001) A genomic study of the bipolar bud site selection pattern in Saccharomyces cerevisiae. Mol Biol Cell 12: 2147-2170. https://doi.org/DOI 10.1091/mbc.12.7.2147

O'Leary MN, Schreiber KH, Zhang Y, Duc ACE, Rao SY, Hale JS, Academia EC, Shah SR, Morton JF, Holstein CA, Martin DB, Kaeberlein M, Ladiges WC, Fink PJ, Mackay VL, Wiest DL, Kennedy $\mathrm{BK}$ (2013) The ribosomal protein Rpl22 controls ribosome composition by directly repressing expression of its own paralog Rpl2211. Plos Genet 9: https://doi.org/ARTNe100370810.1371/journal. pgen.1003708

Pakos-Zebrucka K, Koryga I, Mnich K, Ljujic M, Samali A, Gorman AM (2016) The integrated stress response. EMBO Rep 17: 1374 1395. https://doi.org/10.15252/embr.201642195

Palumbo RJ, Fuchs G, Lutz S, Curcio MJ (2017) Paralog-specific functions of RPL7A and RPL7B mediated by ribosomal protein or snoRNA Dosage in Saccharomyces cerevisiae. G3 (Bethesda) 7: 591-606. https://doi.org/10.1534/g3.116.035931

Paolini NA, Attwood M, Sondalle SB, Vieira C, van Adrichem AM, di Summa FM, O’Donohue MF, Gleizes PE, Rachuri S, Briggs JW, et al (2017) A ribosomopathy reveals decoding defective ribosomes driving human dysmorphism. Am J Hum Genet 100: 506-522. https://doi.org/10.1016/j.ajhg.2017.01.034

Parenteau J, Durand M, Morin G, Gagnon J, Lucier JF, Wellinger RJ, Chabot B, Abou Elela S (2011) Introns within ribosomal protein genes regulate the production and function of yeast ribosomes. Cell 147: 320-331. https://doi.org/10.1016/j.cell.2011.08.044

Parenteau J, Lavoie M, Catala M, Malik-Ghulam M, Gagnon J, Abou Elela S (2015) Preservation of gene duplication increases the regulatory spectrum of ribosomal protein genes and enhances growth under stress. Cell Rep 13: 2516-2526. https://doi.org/10.1016/j.celrep.2015.11.033

Pavitt GD (2018) Regulation of translation initiation factor eIF2B at the hub of the integrated stress response. Wiley Interdisciplinary Reviews-Rna 9: https://doi.org/ARTNe149110.1002/wrna.1491

Pena C, Hurt E, Panse VG (2017) Eukaryotic ribosome assembly transport and quality control. Nat Struct Mol Biol 24: 689-699. https://doi.org/10.1038/nsmb.3454

Quarello P, Garelli E, Carando A, Mancini C, Foglia L, Botto C, Farruggia P, De Keersmaecker K, Aspesi A, Ellis SR, Dianzani I, Ramenghi U (2016) Ribosomal RNA analysis in the diagnosis of Diamond-Blackfan Anaemia. Br J Haematol 172: 782-785. https://doi. org/10.1111/bjh.13880

Ramadori G, Ioris RM, Villanyi Z, Firnkes R, Panasenko OO, Allen G, Konstantinidou G, Aras E, Brenachot X, Biscotti T, Charollais A, Luchetti M, Bezrukov F, Santinelli A, Samad M, Baldi P, Collart MA, Coppari R (2020) FKBP10 regulates protein translation to sustain lung cancer growth. Cell Rep 30: 3851-3863 e3856. https://doi. org/10.1016/j.celrep.2020.02.082

Rao SY, Lee SY, Gutierrez A, Perrigoue J, Thapa RJ, Tu ZG, Jeffers JR, Rhodes M, Anderson S, Oravecz T, Hunger SP, Timakhov RA, Zhang R, Balachandran S, Zambetti GP, Testa JR, Look AT, Wiest DL (2012) Inactivation of ribosomal protein L22 promotes transformation by induction of the stemness factor Lin28B. Blood 120: 3764-3773. https://doi.org/10.1182/blood-2012-03-415349

Reid DW, Nicchitta CV (2015) Diversity and selectivity in mRNA translation on the endoplasmic reticulum. Nat Rev Mol Cell Biol 16: 221-231. https://doi.org/10.1038/nrm3958

Rothman S (2010) How is the balance between protein synthesis and degradation achieved? Theor Biol Med Model 7. https://doi. org/10.1186/1742-4682-7-25

Roux PP, Topisirovic I (2018) Signaling pathways involved in the regulation of mRNA translation. Mol Cell Biol 38: https://doi. org/10.1128/MCB.00070-18

Samir P, Browne CM, Rahul Sun M, Shen BX, Li W, Frank J, Link AJ (2018) Identification of changing ribosome protein compositions using mass spectrometry. Proteomics 18. https://doi.org/ARTN 180021710.1002/pmic.201800217

Santra M, Dill KA, de Graff AMR (2019) Proteostasis collapse is a driver of cell aging and death. Proc Natl Acad Sci U S A 116: 2217322178. https://doi.org/10.1073/pnas.1906592116

Saveanu C, Namane A, Gleizes PE, Lebreton A, Rousselle JC, NoaillacDepeyre J, Gas N, Jacquier A, Fromont-Racine M (2003) Sequential protein association with nascent $60 \mathrm{~S}$ ribosomal particles. Mol Cell Biol 23: 4449-4460. https://doi.org/10.1128/mcb.23.13.44494460.2003

Saxton RA, Sabatini DM (2017) mTOR Signaling in growth metabolism and disease. Cell 169: 361-371. https://doi.org/10.1016/j. cell.2017.03.035
Segev N, Gerst JE (2018) Specialized ribosomes and specific ribosomal protein paralogs control translation of mitochondrial proteins (vol 217 pg 117 2018) J Cell Biol 217: 117-126. https://doi.org/10.1083/ jcb.201706059

Shcherbik N, Pestov DG (2019) The impact of oxidative stress on ribosomes: from injury to regulation. Cells 8: https://doi. org/10.3390/cells8111379

Shi Z, Barna M (2015) Translating the genome in time and space: specialized ribosomes RNA regulons and RNA-binding proteins. Annu Rev Cell Dev Biol 31: 31-54. https://doi.org/10.1146/annurev-cellbio-100814-125346

Shi Z, Fujii K, Kovary KM, Genuth NR, Rost HL, Teruel MN, Barna M (2017) Heterogeneous ribosomes preferentially translate distinct subpools of mRNAs genome-wide. Mol Cell 67: 71-83 e77. https:// doi.org/10.1016/j.molcel.2017.05.021

Shuda M, Velasquez C, Cheng E, Cordek DG, Kwun HJ, Chang Y, Moore PS (2015) CDK1 substitutes for mTOR kinase to activate mitotic cap-dependent protein translation. Proc Natl Acad Sci U S A 112: 5875-5882. https://doi.org/10.1073/pnas.1505787112

Siekevitz P, Palade GE (1958) Heterogeneity of pancreatic ribo-nucleoprotein particles. Fed Proc 17: 311-311

Simsek D, Barna M (2017) An emerging role for the ribosome as a nexus for post-translational modifications. Curr Opin Cell Biol 45: 92-101. https://doi.org/10.1016/j.ceb.2017.02.010

Simsek D, Tiu GC, Flynn RA, Byeon GW, Leppek K, Xu AF, Chang HY, Barna M (2017) The mammalian ribo-interactome reveals ribosome functional diversity and heterogeneity. Cell 169: 1051-1065 e1018. https://doi.org/10.1016/j.cell.2017.05.022

Slavov N, Semrau S, Airoldi E, Budnik B, van Oudenaarden A (2015) Differential stoichiometry among core ribosomal proteins. Cell Reports 13: 865-873. https://doi.org/10.1016/j.celrep.2015.09.056

Sloan KE, Warda AS, Sharma S, Entian KD, Lafontaine DLJ, Bohnsack MT (2017) Tuning the ribosome: The influence of rRNA modification on eukaryotic ribosome biogenesis and function. RNA Biol 14: 1138-1152. https://doi.org/10.1080/15476286.2016.1259781

Spriggs KA, Stoneley M, Bushell M, Willis AE (2008) Re-programming of translation following cell stress allows IRES-mediated translation to predominate. Biol Cell 100: 27-38. https://doi.org/10.1042/ BC20070098

Sriram A, Bohlen J, Teleman AA (2018) Translation acrobatics: how cancer cells exploit alternate modes of translational initiation. EMBO Rep 19. https://doi.org/10.15252/embr.201845947

Sugihara Y, Honda H, Iida T, Morinaga T, Hino S, Okajima T, Matsuda T, Nadano D (2010) Proteomic analysis of rodent ribosomes revealed heterogeneity including ribosomal proteins L10-like L22-like 1 and L39-like. J Proteome Res 9: 1351-1366. https://doi. org/10.1021/pr9008964

Sun L, Yang XW, Chen FF, Li RP, Li XS, Liu ZX, Gu YY, Gong XY, Liu ZH, Wei H, et al (2013) Paralogous ribosomal protein L32-1 and L32-2 in fission yeast may function distinctively in cellular proliferation and quiescence by changing the ratio of Rpl32 paralogs. PLoS One 8. https://doi.org/10.1371/journal.pone.0060689

Tahmasebi S, Khoutorsky A, Mathews MB, Sonenberg N (2018) Translation deregulation in human disease. Nat Rev Mol Cell Biol 19: 791-807. https://doi.org/10.1038/s41580-018-0034-x

Tatsumi K, Yamamoto-Mukai H, Shimizu R, Waguri S, Sou YS, Sakamoto A, Taya C, Shitara H, Hara T, Chung CH, Tanaka K, Yamamoto M, Komatsu M (2011) The Ufm1-activating enzyme Uba5 is indispensable for erythroid differentiation in mice. Nat Commun 2. https://doi.org/10.1038/ncomms1182

Thomson E, Ferreira-Cerca S, Hurt E (2013) Eukaryotic ribosome biogenesis at a glance. J Cell Sci 126: 4815-4821. https://doi. org/10.1242/jcs.111948

Thoreen CC (2017) The molecular basis of mTORC1-regulated translation. Biochem Soc Trans 45: 213-221. https://doi.org/10.1042/ BST20160072

Topf U, Suppanz I, Samluk L, Wrobel L, Boser A, Sakowska P, Knapp B, Pietrzyk MK, Chacinska A, Warscheid B (2018) Quantitative proteomics identifies redox switches for global translation modulation by mitochondrially produced reactive oxygen species. Nat Commun 9: 324. https://doi.org/10.1038/s41467-017-02694-8

Topf U, Uszczynska-Ratajczak B, Chacinska A (2019) Mitochondrial stress-dependent regulation of cellular protein synthesis. J Cell Sci 132: https://doi.org/jcs22625810.1242/jcs.226258

Vajrychova M, Salovska B, Pimkova K, Fabrik I, Tambor V, Kondelova A, Bartek J, Hodny Z (2019) Quantification of cellular protein and redox imbalance using SILAC-iodoTMT methodology. Redox Biol 24. https://doi.org/10122710.1016/j.redox.2019.101227

Walczak CP, Leto DE, Zhang L, Riepe C, Muller RY, DaRosa PA, Ingolia NT, Elias JE, Kopito RR (2019) Ribosomal protein RPL26 is the principal target of UFMylation. Proc Natl Acad Sci U S A 116: 1299-1308. https://doi.org/10.1073/pnas.1816202116

Wang L, Xu Y, Rogers H, Saidi L, Noguchi CT, Li H, Yewdell JW, Guydosh NR, Ye Y (2020) UFMylation of RPL26 links translocation-associated quality control to endoplasmic reticulum protein ho- 
meostasis. Cell Res 30: 5-20. https://doi.org/10.1038/s41422-019_ 0236-6

Wapinski I, Pfiffner J, French C, Socha A, Thompson DA, Regev A (2010) Gene duplication and the evolution of ribosomal protein gene regulation in yeast. Proc Natl Acad Sci U S A 107: 5505-5510. https://doi.org/10.1073/pnas.0911905107

Wei Y, Xu X (2016) UFMylation: A unique \& fashionable modification for life. Genomics Proteomics Bioinformatics 14: 140-146. https:// doi.org/10.1016/j.gpb.2016.04.001

Wek RC, Jiang HY, Anthony TG (2006) Coping with stress: eIF2 kinases and translational control. Biochem Soc Trans 34(Pt 1): 7-11. https://doi.org/10.1042/BST20060007

Wolfe KH, Shields DC (1997) Molecular evidence for an ancient duplication of the entire yeast genome. Nature 387: 708-713. https:// doi.org/10.1038/42711

Wong QWL, Li J, Ng SR, Lim SG, Yang H, Vardy LA (2014) RPL39L is an example of a recently evolved ribosomal protein paralog that shows highly specific tissue expression patterns and is upregulated in ESCs and HCC tumors. RNA Biol 11: 33-41. https://doi. org/10.4161/rna.27427

Wrobel L, Topf U, Bragoszewski P, Wiese S, Sztolsztener ME, Oeljeklaus S, Varabyova A, Lirski M, Chroscicki P, Mroczek S,
Januszewicz E, Dziembowski A, Koblowska M, Warscheid B, Chacinska A (2015) Mistargeted mitochondrial proteins activate a proteostatic response in the cytosol. Nature 524: 485-488. https://doi. org $/ 10.1038 /$ nature14951

Xue S, Barna M (2012) Specialized ribosomes: a new frontier in gene regulation and organismal biology. Nat Rev Mol Cell Biol 13: 355369. https://doi.org/10.1038/nrm3359

Yusupova G, Yusupov M (2017) Crystal structure of eukaryotic ribosome and its complexes with inhibitors. Phil Trans Royal Soc B-Biol Sci 372. https://doi.org/10.1098/rstb.2016.0184

Zhang Y, Xu H (2016) Translational regulation of mitochondrial biogenesis. Biochem Soc Trans 44: 1717-1724. https://doi.org/10.1042/ BST20160071C

Zhou Y, Dong F, Lanz TA, Reinhart V, Li M, Liu L, Zou J, Xi HS, Mao Y (2018) Interactome analysis reveals ZNF804A a schizophrenia risk gene as a novel component of protein translational machinery critical for embryonic neurodevelopment. Mol Psychiatry 23: 952-962. https://doi.org/10.1038/mp.2017.166

Zhou Y, Musalgaonkar S, Johnson AW, Taylor DW (2019) Tightlyorchestrated rearrangements govern catalytic center assembly of the ribosome. Nat Commun 10. https://doi.org/10.1038/s41467-01908880-0 